\title{
Development, verification and application of numerical model for coupled free surface and liquid metal flow calculation in EM field
}

\author{
S. Spitans ${ }^{1,2}$, A. Jakovics ${ }^{2}$, E. Baake ${ }^{1}$ \& B. Nacke ${ }^{1}$ \\ ${ }^{1}$ Institute of Electrotechnology, Leibniz University, Germany \\ ${ }^{2}$ Laboratory for Mathematical Modelling of Environmental and \\ Technological Processes, University of Latvia, Latvia
}

\begin{abstract}
On account of ANSYS Classic, ANSYS CFX and ANSYS Fluent external coupling a numerical model for EM induced flow and free surface dynamics of melt in induction furnaces is developed. The model is adjusted for the case of EM levitation and extended on 3D with application of LES turbulence.

Calculated steady state free surface shapes are compared to other models and experimental measurements in induction furnaces and EM levitation melting setup, predicted free surface dynamics is compared to analytical estimation of free surface oscillation period. The model accuracy is approved.

The model is applied for tailoring EM levitation furnace design to meet conditions for levitation of greater charge weight. Numerical results reveal difficulties that must be considered in case of EM levitation in horizontal orthogonal EM field and appear to be in a good agreement with experiment.

Keywords: numerical simulation, EM induction furnace, EM levitation, twophase turbulent flow, free surface.
\end{abstract}

\section{Introduction and former research}

Induction furnaces that ensure contact less control of EM stirring, molten metal free surface and temperature are widely applied in metallurgical industry. Requirements for the free surface shape and behaviour are dedicated by the different tasks of particular technological process. Meanwhile, the dynamics of free surface might be complicated by the reciprocal interaction between free 
surface shape, EM field and flow pattern, as well as notably unsteady due to the switch between the furnace power regimes, mean flow instability and turbulence.

Since free surface control is significant for processing of metallic materials, the development of models that consider free surface dynamics remains relevant.

Advanced multiphysical processes like energy and mass transfer, crystallization and homogenization of alloying particles are calculated nowadays in 3D with fixed hydrostatic steady free surface shape and précised LES turbulence [1].

Free surface dynamics of EM levitated melt, flow and energy transfer in 2D consideration, as well as crystallization processes with free surface behaviour in EM induction furnaces were successfully simulated using simplified twoparameter turbulence models [2].

The results of 3D numerical calculation of liquid droplet dynamics in a high DC magnetic field were published recently [3].

However, at the present moment there is no approach developed for 3D calculation of multiphysical processes in EM induction equipment with consideration of free surface dynamics and application of LES description for turbulent flow. The previous investigations revealed that in case of ICF with two characteristic mean flow vortexes only the LES model gives comparable results to experimental measurements [1].

The free surface oscillation period in axisymmetric ICF is estimated analytically [4]. In this simplified case the Lorentz force is considered radial and constant and the typical oscillation period $T$ is fully dependent on the crucible geometry (1)

$$
T_{\text {theor }}=2 \pi r_{0}^{0.5}\left(\lambda_{1} \cdot g\right)^{-0.5} \cdot \tan \left(\lambda_{1} \cdot h_{0} / r_{0}\right),
$$

$r_{0}$ is crucible radius, $h_{0}$ is initial filling and $\lambda_{1}=3.83$ - Bessel function $J_{1}$ solution.

The results of eq. (1) validation by comparison to experimental data exposed a good agreement for the case of small amplitude oscillations [4].

\section{Model formulation [5]}

\subsection{Model assumptions}

Due to harmonic EM field and induced currents, the Lorentz force can be decomposed into a steady and harmonic part that oscillates with double frequency

$$
\vec{f}_{\text {Lor }}=\left\langle\vec{f}_{\text {Lor }}\right\rangle+\overrightarrow{\tilde{f}}_{\text {Lor }} \cdot \cos (2 \omega t+\varphi),
$$

where $\omega$ is an angular frequency of EM field and $\varphi$ is a phase.

Because of greater inertia times of melt in comparison to the EM field timescale $(\omega /(2 \pi)>50 \mathrm{~Hz})$, only the steady part of the Lorentz force is taken into account.

Let us consider the non-dimensional frequency $\hat{\omega}$ that shows the relation between the induced and external EM field, and magnetic Reynolds number $R e_{m}$ that gives the ratio of the flow generated and external EM field. 


$$
\hat{\omega}=\omega \sigma \mu_{0} r_{0}^{2} \text { and } R e_{m}=\sigma \mu_{0} r_{0} v_{0} \text {. }
$$

Combining $\hat{\omega}$ and $R e_{m}$ it can be shown that in typical case of induction furnace

$$
\hat{\omega} / R e_{m}=\omega r_{0} / v_{0}>>1, \quad \omega /(2 \pi)>50 \mathrm{~Hz}, \quad r_{0} \approx 1 \mathrm{~m}, \quad v_{0} \approx 1 \mathrm{~m} / \mathrm{s}
$$

generated EM field by the flow is insignificant in comparison to induced one. Assuming no free charge in the system and neglecting displacement currents (no EM wave radiation) the reduced Maxwell equation system in addition with simplified Ohms law (no EM field generation by the flow) is used for harmonic analysis in ANSYS Classic and the Lorentz force distribution in melt at particular instant free surface shape is obtained.

In the HD part of calculation the Navier-Stokes equation for incompressible fluid is solved in ANSYS Fluent. In typical cases of ICF the Reynolds number

$$
R e=r_{0} v \rho / \eta>10^{3}
$$

indicates on developed turbulent flow, thus k- $\omega$ SST or LES turbulence is used. Volume of Fluid (VOF) numerical technique is applied for calculation of twophase flow dynamics. In VOF technique the phase distribution is represented with a scalar volume fraction field $F\left(x_{i}, y_{i}, z_{i}, t\right)$. In particular case, $F=1$ when mesh element contains only primary phase (melt) and $F=0$ when element contains secondary phase (air). When phase surface crosses element $-0<F<1$. For phase dynamics additional transport equation is solved

$$
\partial F / \partial t+\vec{v} \cdot \nabla F=0
$$

and free surface is reconstructed as isosurface of $F=0.5$.

Volume density of surface tension force is calculated as

$$
\vec{f}_{\gamma}=-\gamma(\nabla \cdot \vec{n}) \vec{n} \delta_{\gamma}
$$

where $\gamma$ is surface tension coefficient, $\vec{n}$ is free surface normal and $\delta_{\gamma}$ is Delta function that ensures that surface tension force is located only at free surface.

\subsection{Model technical implementation}

Calculation of free surface dynamics of EM induced metal flow is arranged by means of ANSYS Classic for EM calculation, ANSYS Fluent for two-phase flow calculation, ANSYS CFX for post-processing and external coupler - a batch file.

Initial free surface shape of molten metal, as well as every instant shape obtained with HD calculation is written into a file. This file contains free surface keypoint (KP) numbers, KP coordinates and series of KP number sequences that indicate the order of free surface KP connection for definition of elementary polygons.

Transferring free surface KPs and elementary polygons from CFX-Post to ANSYS Classic a self written filtering procedure is performed in order to avoid generation of degenerate surface polygons that have great edge length ratios and cause problems in ANSYS Classic volume mesher. Hence, filtered free surface 
consisting of elementary triangular non-degenerate areas is obtained and the finite element mesh for EM problem is constructed.

Then the distribution of harmonic EM field is calculated for the fixed free surface shape. The coordinates of alloy mesh element centroids, as well as the values of Lorentz force density components, are retrieved and written into a file.

In the beginning of the transient HD calculation the Lorentz force density is interpolated on the Fluent finite volume mesh and used as mechanical momentum source in two-phase flow equations. Then the calculation of unsteady flow is performed for sufficiently small time interval for which the slight change of free surface shape is insignificant for the Lorentz force distribution.

Unphysical air acceleration due to inevitably diffused interface in VOF formulation is damped by regular air velocity reinitialization in a small distance from the melt free surface. Such technical trick allowed us to ensure a stable calculation of free surface dynamics for the case of highly pronounced EM skineffect.

By the end of HD calculation a new transient free surface state is obtained and written into a file. The recalculation of Lorentz force distribution upon the new free surface shape is performed further and the repeat of the whole calculation loop ensures fully automatic free surface dynamics computation.

\section{Model verification}

\subsection{D calculation of steady state free surface in ICF}

The first verification of developed numerical approach is performed by comparison between a steady state meniscus shape experimental measurements in big laboratory scale ICF $[1,5]$ our 2D calculation results and results of 2D steady simulation of Pesteanu and Baake [6] (fig. 1(a)).

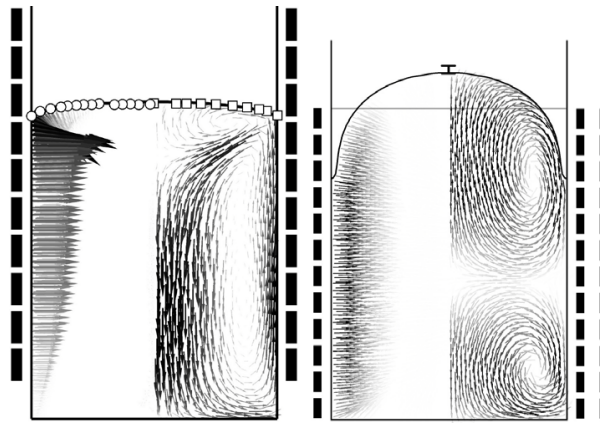

(a) (b)

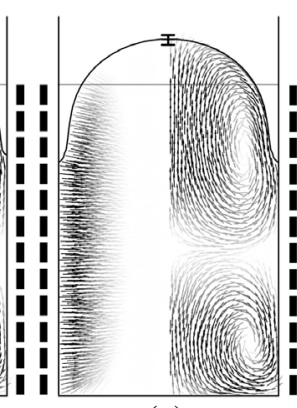

(c)

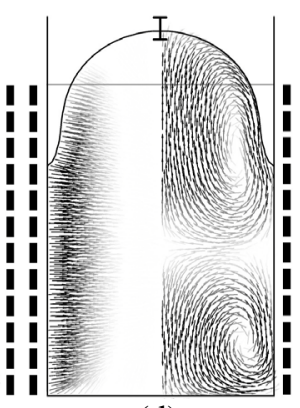

(d)

Figure 1: $\quad$ Lorentz force distribution (left), steady flow (right) and meniscus shape obtained by 2D model in comparison to (a) - experimental measurements [1] (circles - on the left) and calculation [6] (squares - on the right) in big ICF and (b), (c), (d) - experimental measurements of meniscus heights (ticks) above initial filling (horizontal line) in small ICF at three different power regimes. 
Distribution of the Lorentz force $\left(0-0.1 \mathrm{MN} / \mathrm{m}^{3}\right)$, steady flow $(0-25 \mathrm{~cm} / \mathrm{s})$ with characteristic two torroidal vortex and calculated meniscus shape in comparison to experiment [1] and 2D calculation [6] proves a good agreement obtained.

The weak point of this verification is insignificant meniscus height in comparison to initial filling. Imprecision in geometry or material properties might lead to insensible deviations of meniscus shape in respect to initial filling that in the scale of meniscus height can reach several times.

Hence, experimental measurements of meniscus heights above initial filling (by E. Baake) in small laboratory scale ICF [5] were used for the next verification of model. Due to the smaller furnace size and greater inductor turn linear density it was possible to achieve greater power densities that ensured prominent melt squeeze by the EM field and greater meniscus heights in respect to initial filling.

Calculated quasi steady flow pattern $(0-55 \mathrm{~cm} / \mathrm{s})$ and Lorentz force density distribution (0-2.5 $\left.\mathrm{MN} / \mathrm{m}^{3}\right)$, as well as meniscus shape in comparison to the experimental data of meniscus height above initial flat free surface are shown in fig. 1, (b)-(d). Experimental measurements are marked by the ticks, while the ticks vertical lines correspond for the measurement errors due to the turbulence and mean flow instability that caused free surface fluctuations.

The calculated meniscus is in good agreement with experiment and obtained discrepancies are below the measurement error. Regardless of meniscus heights are comparable to initial filling these experiments are unable to show the agreement between the real and calculated meniscus shape. Therefore, experimental measurements of highly pronounced meniscus shapes were used for the further model verification.

\subsection{Quasi 3D calculation of steady state free surface shape in IFCC}

Experimental measurements of aluminum melt free surface shape in industrial scale IFCC were used [5, 7]. The furnace model consisted of copper crucible wall divided on 26 sections with a short circuit ring in the lower part, unsectioned copper bottom and copper inductor with five turns (fig. 2(a)).

On account of the symmetry of setup the EM calculation was performed only for one section considering azimuthal inhomogeneity of EM field due to the sectioned crucible (fig. 2(b)). Air gap of $1 \mathrm{~mm}$ was ensured between the melt, the bottom and the crucible walls due to the great electrical resistivity of the skull that appears in the contact regions between the melt and water-cooled crucible.

Meanwhile, the HD calculation was performed on a mesh with one element resolution of section in azimuthal direction that ensured azimuthal averaging of the Lorentz force for the 2D axisymmetric approximation.

The comparison between the measured [7] and calculated free surface shapes of melt in IFCC at different initial fillings and power regimes revealed a fine correlation between the model prediction and experiment (fig. 2, (c)-(g)). Measured and calculated free surface profiles, as well as Lorentz force distribution and velocity patterns sketch the good agreement for performed comparison and approve accuracy of developed numerical approach. 


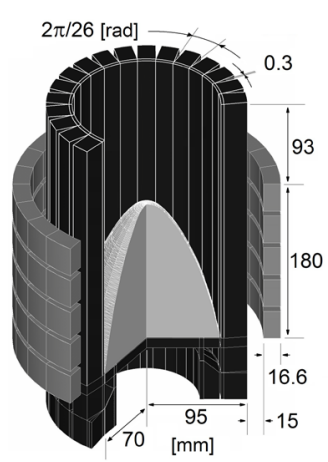

(a)

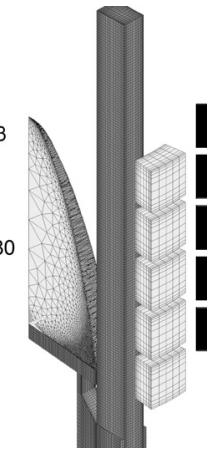

(b)

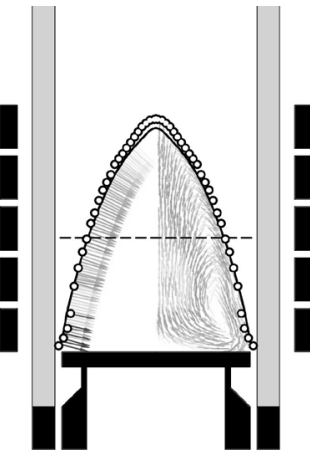

(c)

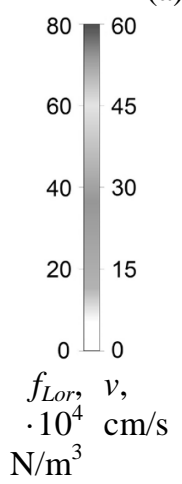

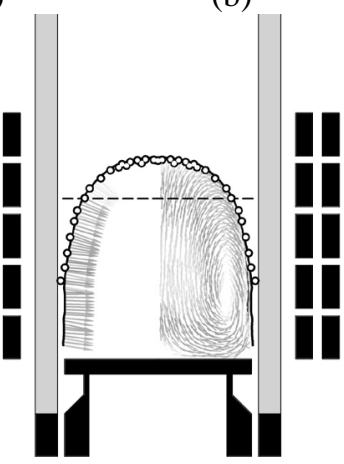

(e)

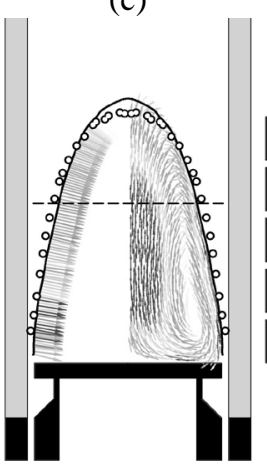

(f)

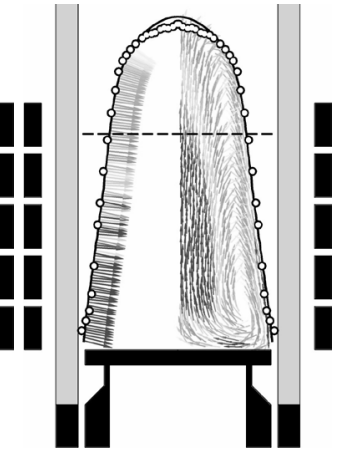

(d)

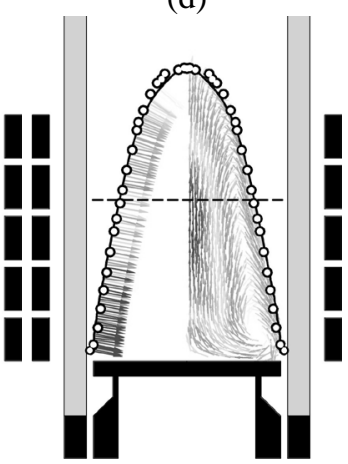

(g)

Figure 2: $\quad$ Geometry of IFCC with sectioned crucible [7] (a) and mesh for EM calculation (b). As well as measured (points) [7] and calculated (bold line) steady free surface shapes, Lorentz force distributions (on the right) and steady flow patterns (on the left) for different initial fillings (dashed line) and power regimes.

\subsection{Analytic verification of free surface oscillation period in ICF}

At the moment no experimental data is available for validation of calculated free surface dynamics in ICF, however, numerical results can be compared to analytical estimation of small amplitude free surface oscillations (1) [4].

For this purpose an industrial type ICF adopted from [4] with crucible that is already filled with molten aluminum is considered and it is assumed that at initial time moment of $t=0 \mathrm{~s}$ the furnace instantly reaches its operating state.

The dynamics of free surface profile sketches free surface regular oscillations and proves that the discrepancy between the numerically obtained oscillation period $T_{\text {calc }}=0.68 \mathrm{~s}$ and analytical approximation of small deviations $T_{\text {theor }}=$ $0.676 \mathrm{~s}$ is less than $1 \%$. Moreover, the calculated instant free surface states are in good qualitative agreement with calculation [4] (fig. 3(a)). 


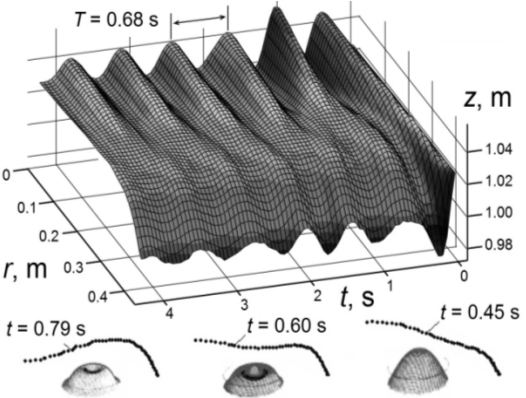

(a)

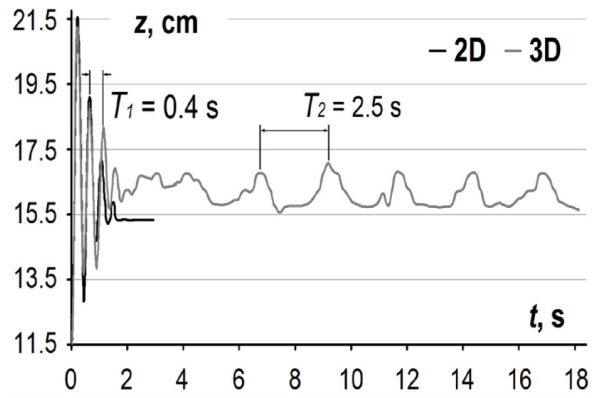

(b)

Figure 3: (a) 2D calculation results for free surface profile dynamics in industrial type ICF and meniscus shape comparison to calculation [4]; (b) free surface point oscillations along cylindrical crucible $z$ axis obtained with 2D and 3D models in big ICF with $I_{e f}=5 \mathrm{kA}$.

For the further validation big ICF from [1] is considered. It is assumed that cylindrical crucible is filled with melt (zero velocity and flat free surface) and at $t$ $=0 \mathrm{~s}$ inductor is instantly fed with AC current of $I_{e f}=5 \mathrm{kA}$.

The first $2 \mathrm{~s}$ of flow calculated with 2D and 3D models (fig. 3(b)) indicate on similar behavior of free surface point oscillations on the symmetry axis. Moreover, the period of these damping free surface oscillations $\left(T_{1}=0.4 \mathrm{~s}\right)$ is in good agreement with analytical estimation $\left(T_{\text {theor }}=0.39 \mathrm{~s}\right)$ according to (1) [4].

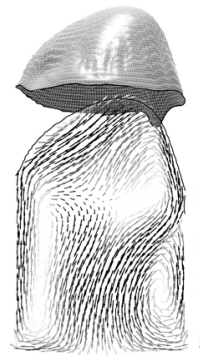

$t=10.2 \mathrm{~s}$
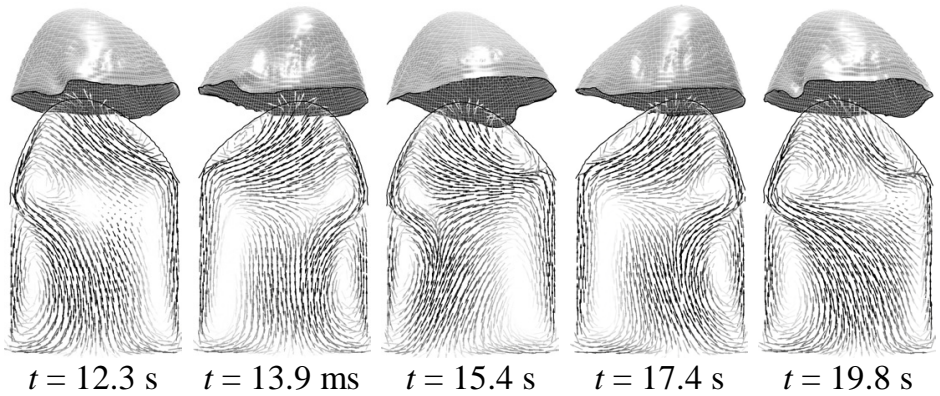

Figure 4: Instant flow patterns (0-0.75 m/s) and free surface shapes obtained with $3 \mathrm{D}$ calculation for the case of big ICF operating at $I_{e f}=7 \mathrm{kA}$.

The next $4 \mathrm{~s}$ correspond for the flow transition to a steady state and approximately from $t=6 \mathrm{~s}$ the developed turbulent flow starts to oscillate with periodical reallocation of torroidal vortexes. Free surface shape, EM field and melt flow interaction leads to meniscus staggering with a period of $T_{2}=2.5 \mathrm{~s}$ (fig. 3(b)).

Higher meniscus height and maximal velocity values, as well as the same low frequency quasi steady state free surface oscillations, are obtained for the case of greater inductor current $I_{e f}=7 \mathrm{kA}$ in big ICF (fig. 4). 
Model accuracy for the free surface small amplitude oscillations has been proved. Investigations of meniscus low frequency oscillations at a quasi steady state regime in ICF form further plans of research.

\subsection{D calculation of steady state free surface shape in single-frequency EM levitation melting furnace}

The laboratory scale single-frequency EM levitation melting furnace with a pair of water-cooled coaxial inductor coils and a ferrite yoke is considered [6]. Quartz tube is placed in the air gap between yoke ends and inductor coils in order to prevent the unexpected contact between the molten metal and furnace parts. For particular experimental configuration the isosurface of magnetic induction is saddle-shaped with local minimum at $z=0$ along $z$ axis and local maximum at $x$ $=0$ along $x$ axis. Hence, the position of EM levitated sample is unstable and it is pushed to the quartz tube wall (fig. 5(a)). In order to stabilize the sample on the $y$ axis four magnetic material teeth are introduced along yoke ends (fig 5(b)).

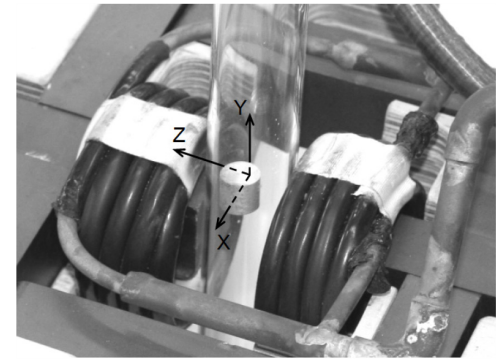

(a)

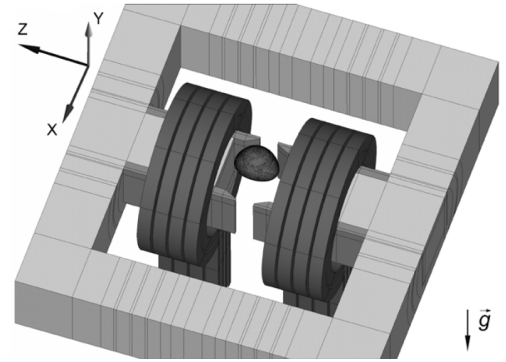

(b)

Figure 5: $\quad$ EM levitation of solid aluminum cylinder that touches quartz tube walls in EM levitation melting setup without additional magnetic material teeth [6] (a) and model of modified setup with introduced magnetic material teeth and stabilized molten aluminum (b).

In the beginning of calculation the drop is given a spherical shape and zero velocity. It is placed a few millimeters above its experimentally observed state.

The comparison between 3D calculation results, experimental measurements [6] and 2D steady calculation [6] are presented in fig. 6. It can be noticed that droplet shapes obtained with both models are in good agreement with experiment. Lorentz force distribution and steady flow pattern on cross-sections illustrate the quasi steady state in 3D calculation of EM levitation (fig. 6).

The qualitative comparison between experiment photo [6] and picture of numerical model also reveals good agreement for the droplet shape (fig. 7).

The nature of the droplet shape is analyzed. Alternate current in inductor generates alternate EM field that due to the great magnetic permeability of ferrite is mainly concentrated in the yoke. In the air gap region magnetic field lines spread and due to the skin effect flow around conductive droplet from one yoke end to another. In the regions where magnetic field lines separate at the surface 


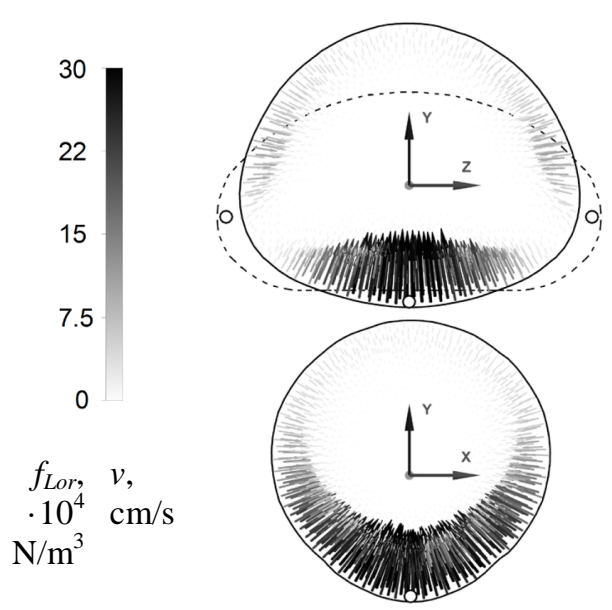

(a)

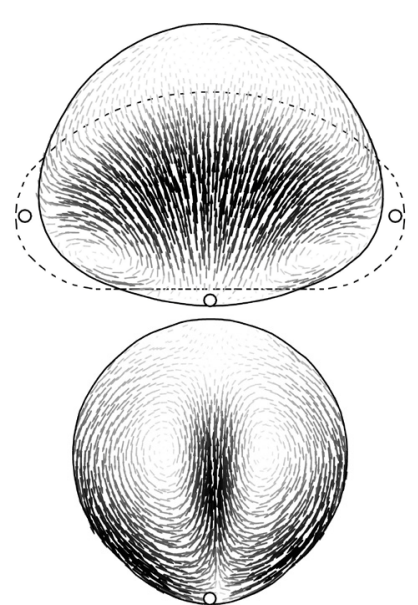

(b)

Figure 6: $\quad 3 \mathrm{D}$ calculation results for (a) Lorentz force distribution, (b) steady flow pattern and free surface shape (bold line) in comparison to 2D calculation [6] (dashed line) and experimental measurements (circles).

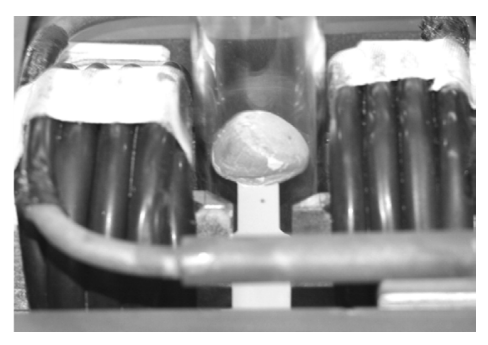

(a)

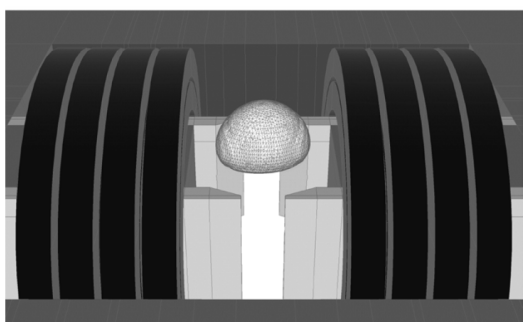

(b)

Figure 7: Qualitative comparison between (a) experimentally observed [6] and (b) numerically simulated levitation in single-frequency EM field.

of the drop (singularity regions) the minimum of Lorentz force is expected due to the small magnetic field component parallel to the free surface. Meanwhile, maximum of Lorentz force is obtained at the bottom of the drop due to the greater field intensity and dominating field component along free surface. The following Lorentz force distribution contributes to the stretching of the drop along magnetic field lines. In some time curvature radiuses of droplet singularity regions become small enough and growing contribution of surface tension effects will stop the droplet stretching.

For the greater masses of levitated droplets the particular single-frequency horizontal EM field will contribute to the droplet shapes that are stretched along 
EM field lines. In the meantime, the length of the droplet is limited due to the diameter of quartz tube, distance between inductor coils or yoke ends.

\section{Model application for EM levitation scale-up}

In order to increase the EM levitated droplet weight, it is considered to install additional orthogonal horizontal EM field by means of the technical realization presented in fig 8 . The modified setup consists of two separate pairs of watercooled coaxial inductor coils with orthogonal axis orientation that generate horizontal and perpendicular EM fields. In order to obtain greater magnetic induction in the region of levitating droplet the EM field is conducted by the yoke ends and looped in the outer yoke. Magnetic material teeth are arranged along yoke ends in order to redistribute the EM field and stabilize molten metal sample on $z$ axis.

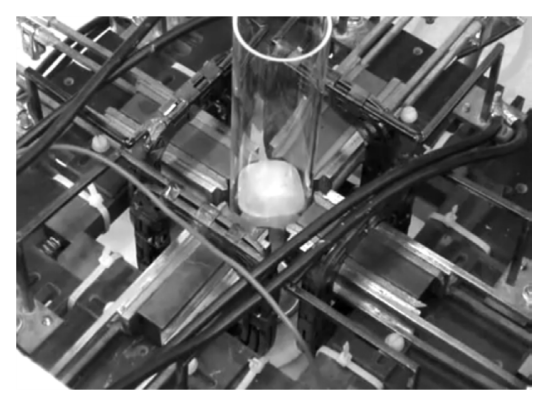

(a)

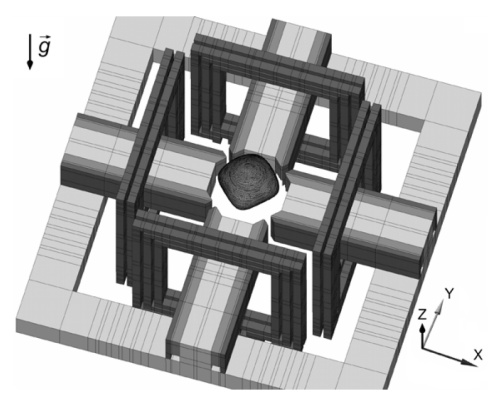

(b)

Figure 8: (a) Experimental installation [6] and (b) numerical model of EM levitation melting setup with two orthogonal horizontal EM fields.

In the beginning of simulation the molten aluminum droplet ( $m=33 \mathrm{~g}$ ) has a spherical shape and zero velocity. Both inductor coil pairs are fed with an alternate current of the same frequency and two marginal cases are considered: the phase shift between coaxial coil pairs is $\varphi=0$ and $\varphi=\pi / 2$.

When the phase shift between coaxial coil pairs is $\varphi=0$ the generated EM field has only one dominant component along $x=-y$ which determines the droplet stretching in the same direction (fig. 9(a)). The simulation shows that for increased droplet mass the length of stretched droplet is also enlarged and curvature radius of two singularity regions reduced, because the melt leakage in these regions is still prevented mainly by the surface tension. This leads to an undesirable situation when the droplet touches the wall of quartz tube.

In the second case, when the phase shift between coaxial coil pairs is $\varphi=\pi / 2$ the clockwise rotational EM field is obtained. Despite the azimuthal component of induced Lorentz force is small in comparison to radial and axial components the velocity of flow increases rapidly and at $t=70 \mathrm{~ms}$ azimuthal velocity already reaches the value of $v=1.5 \mathrm{~m} / \mathrm{s}$ at free surface. With such rapidly increasing rotation due to the centrifugal force the droplet radius in $x y$ plane grows and it 


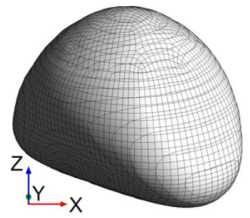

$t=20 \mathrm{~ms}$

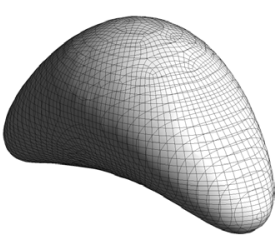

$t=35 \mathrm{~ms}$

(a)

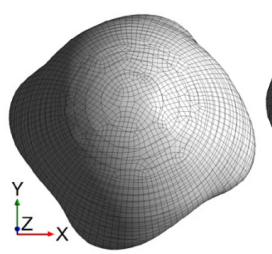

$t=38 \mathrm{~ms}$

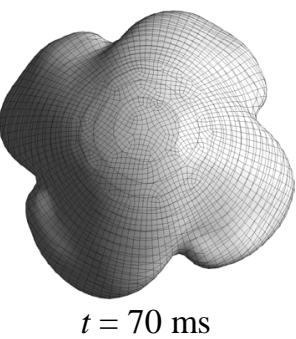

(b)

Figure 9: Dynamics of molten aluminum shape in two-frequency EM levitation melting setup when $f_{1}=f_{2}$ and a phase shift between orthogonal inductors is $\varphi=0$ (a) and $\varphi=\pi / 2$ (b) (clockwise rotational field).

leads also to undesirable contact between the droplet and the quartz tube wall (fig. 9(b)). The droplet gains such great rotational velocity because it starts to rotate as a solid body and during this rotation it loses momentum only because of friction with air that has several magnitudes lover viscosity and density. Meanwhile, when there is no sensible azimuthal Lorentz force component the flow can have azimuthal velocity comparable to the radial and axial velocity components that are limited due to the molecular and turbulent viscosity of liquid metal.

In order to levitate greater charge mass the EM fields of two non-interacting frequencies $f_{1}$ and $f_{2}$ must be applied for the case of setup presented in fig. 8 [6].

3D numerical results of the Lorentz force and steady flow on cross-sections of the drop in two-frequency EM levitation furnace are obtained (fig. 10).
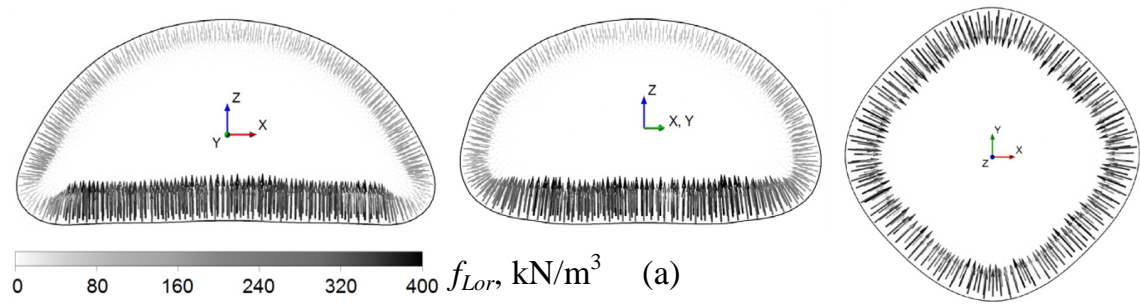

$f_{\text {Lor }}, \mathrm{kN} / \mathrm{m}^{3}$

(a)
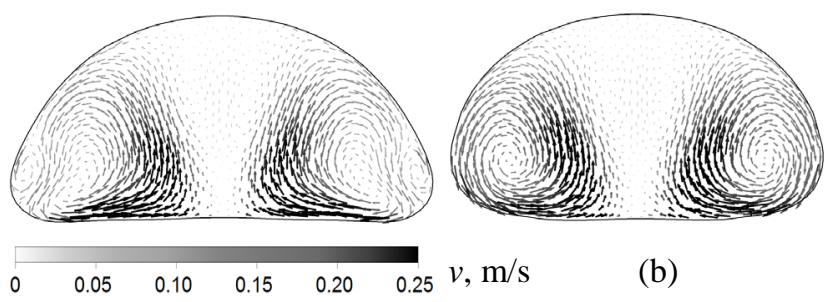

(b)

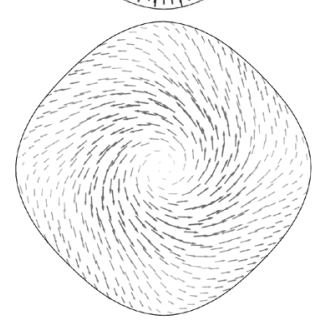

Figure 10: $3 \mathrm{D}$ calculation results on cross-sections of molten aluminum drop for free surface shape, Lorentz force distribution (a) and steady flow (b) in two-frequency EM levitation melting setup. 


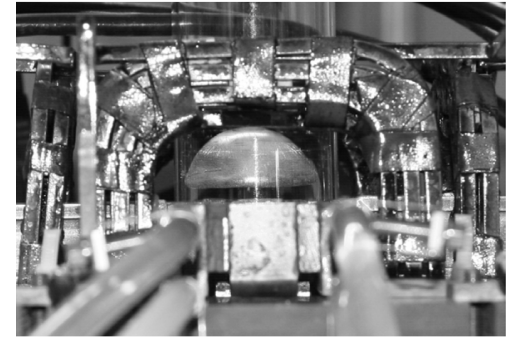

(a)

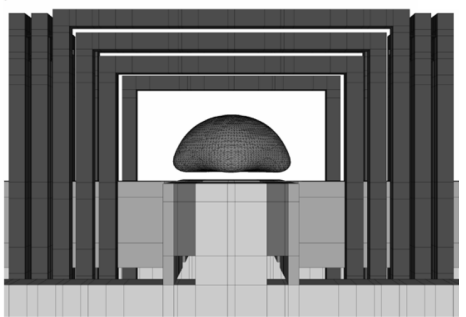

(b)

Figure 11: Comparison between (a) experimentally observed [6] and (b) numerically simulated EM levitation.

From the distribution of the Lorentz force it can be clearly noticed that in particular orthogonal non-interacting two-frequency EM field the undesirable singularity of force in the regions of EM field line separation is reduced, hence smaller droplet dimensions are obtained in $x y$ plane. The stability of the quasi steady state shape over time, the flat bottom of droplet and a "square" profile in $x y$ plane is in good correlation with experiment. Meanwhile, the distribution of the Lorentz force, complete force domination at the bottom and local maximums on the top surface right above singularity regions, contributes to a formation of one large torroidal vortex and a tiny flow recirculation (fig. 10(b), xz plane). Moreover, despite that the Lorentz force has no sensible azimuthal component the flow in $x y$ plane arranges itself in one slow vortex around $z$ axis. Presumably, this effect is caused by asymmetry introduced by consideration of inductor coils of a spiral shape, however, requires a deeper investigation.

The comparison between experiment photo and picture of numerical model also reveals a good agreement for the levitating droplet shape (fig. 8 and fig. 11).

\section{Conclusions}

2D model for coupled free surface and liquid metal flow numerical calculation in EM field is developed. The model is adjusted for the case of EM levitation, extended on 3D consideration and can be used with LES turbulence approach.

The comparison of our k- $\omega$ SST calculation results to appropriate experimental measurements and results of other models for the steady state free surface in induction furnaces at different regimes and EM levitation melting setup, as well as comparison of free surface oscillation period to analytical estimation, revealed good correlation and approved accuracy of implemented model.

Using experimental data and developed numerical approach the new method for drip- and leakage-free EM levitation melting of metallic samples with greater weights and stabilized positions proposed by Pesteanu et al. [6] has been successfully validated. It is planned to tailor the design of setup and configuration of EM field in order to meet the conditions for stable EM levitation of industrial-scale molten metal charge and reproduce it in experiment. 


\section{Acknowledgements}

Current research was performed with financial support of the ESF project of the University of Latvia, contract No. 2009/0138/1DP/1.1.2.1.2/09/IPIA/VIAA/004. The authors thank the German Research Association (DFG) for supporting this study under the Grant No. BA 3565/3-1. The authors would like to acknowledge the great scientist Prof. Ovidiu Pesteanu (*1945-†2012) for development of particular EM levitation technology, contribution and support in this research.

\section{References}

[1] Kirpo, M. Modelling of turbulence properties and particle transport in recirculated flows. Ph.D. Thesis, University of Latvia, Riga, 2008.

[2] Bojarevics, V., Harding, R., Pericleous, K. and Wickins, M. The development and experimental validation of a numerical model of an induction skull melting furnace. Met. Mat. Trans. B Ser., 35, pp. 785-803, 2004.

[3] Easter, S., Bojarevics, V. and Pericleous, K. Numerical modelling of liquid droplet dynamics in microgravity. J. Phys.: Conf. Ser., 327 012027, 2011.

[4] Hegewaldt, F., Buligins, L. and Jakowitsch, A. Transient bath surface bulging at energization of an ind.-type CF. Elektrowärme int., 1, pp. 28-42, 1993.

[5] Spitans, S., Jakovics, A., Baake, E. and Nacke, B. Numerical modelling of free surface dynamics of melt in an alternate EM field. Part I: implementation and verification of model. Met. Mat. Trans. B Ser., DOI: 10.1007/S11663-013-9809-9, pp. 1-13, 2013.

[6] Pesteanu, O. and Baake, E. The multicell VOF method for free surface simulation of MHD flows. Part I: Mathematical model and Part II: Experimental verifications and results. ISIJ International, 51(5), pp. 707721, 2011.

[7] Westphal, E. Elektromagnetisches und thermisches Verhalten des KaltwandInduktions-Tiegelofens. Dr-Ing. Dissertation, Dusseldorf, 21(210), 1996. 